\title{
The Development and Application of Virtual System of Physiological Function Experiments Based on Networks
}

\author{
Deli Wang \\ College of Life Sciences, Jilin University \\ Changchun ,130012, China \\ e-mail:wangdl@jlu.edu.cn \\ Haifeng Tang, Xiaodong Ren \\ College of Life Sciences, Jilin University \\ Changchun ,130012,China \\ e-mail:tanghf@jlu.edu.cne-mail:renxd@jlu.edu.cn
}

\begin{abstract}
Purpose]To inspire students' interest in learning, cultivate their spirit of innovation, and comprehensively improve the level of experimental lesson, the virtual system of physiological function experiments based on networks was developed, applied. [Methods] With establishment of the data management and service functions, and the support of the database, this system provides material library for various functional virtual experiments It could respond the client's access successfully. In addition, the system is available for campus network and internet access with employing network architecture. [Results] Through the actual operation, it is shown that the system run stably and well with vivid images. It is conducive to stimulate students' initiative and develop their ability to innovate. [Conclusions] This system can be used in classroom, or for self-studying with networks. Therefore, it could enhances the students' interest in learning and improves their creativity and imagination.
\end{abstract}

Keywords-networks;physiology;real teaching; virtual experimen

\section{INTRODUCTION}

The rapid development of the increasing popularity of computer and network technology inevitably brings new and greater development space for experimental teaching reform of higher education ${ }^{[1]}$. Physiology is an important basic course of medicine and life sciences, moreover , physiological function experiment is an important part of the physiology and indispensable ways of teaching. Virtual experimental system consists of virtual experiments based on computer simulation technology and network structure, etc.

According to the basic requirements of physiological function experiments, the virtual technology in computer systems have various virtual experiment environments, the experiment instruments and equipments as real environment. In addition, the experimental models can be operated in real time ${ }^{[2,3]}$ while various predetermined experimental projects can be completed. It is shown the simulation training has better effect than that in real environment.

In recent years, due to the significant increase in college enrollment students compared with lack of faculty, experimental conditions, funding and class hour, the experimental lesson has been extremely restricted. Even some experimental projects are unable to carry out, which

\author{
Kun Fu \\ Office of Teaching Affairs, Jilin University \\ Changchun, 130012, China \\ e-mail:fukun@jlu.edu.cn \\ Qingfan Meng ,Lirong Teng \\ College of Life Sciences, Jilin University \\ Changchun,130012,China \\ e-mail:mengqf@jlu.edu.cn e-mail:tenglr@jlu.edu.cn
}

lead to the integrity and systemic of physiology lesson being declined. It is a good idea to use computer network simulation technology for experimental teaching reform. Through virtual experiments introduced to the physiology experiments, students could understand the experimental principles and steps better. With the formal experimental operation and courseware, students can experience physiological experiments virtually which could expand students' knowledge ${ }^{[4]}$. Paperless examination can be achieved at the same time. The process achieves low carbon, energy-saving, reducing teacher workload, improving work efficiency and saving students' limited class time which gives students more time to complete experiments better. In addition, it could improve students' ability, cultivate their innovative ${ }^{[5]}$, and improve the level of experimental teaching.

\section{FUNCTIONAL CHARACTERS OF VIRTUAL EXPERIMENTAL SYSTEM OF PHYSIOLOGICAL FUNCTION}

According to the requirements of lesson of physiological function experiments vivid physiology experiments environment can be provided. The instruments and equipments can be used and operated easily. For specific experiments, the entire process can be simulated by the experimental model with real-time computer simulation technology ${ }^{[6]}$. Various data generated can be recorded and analyzed in real-time. The system has other auxiliary functions needed, such as experimental purposes, principles and requirements, laboratory instruments, equipment introduction, etc. Students can choose the experimental animals, the experimental equipment and processing factors to complete experimental design and experiment.

The system uses a virtual simulation and network technology, which cover a large number of functional experiment without experimental animals and experimental preparation. It could help students understand the experimental steps and experimental results as a useful functional experiment teaching supplement. It has the following advantages:

1) Simulation: The virtual reality technology was used to construct a simulation experiment environment. 
2) Interaction: The multimedia programming software was used to design the experiment, which resulted real-time human-computer interaction.

3) interesting innovation: It simulated interesting and allowed students to have arbitrary choice of experimental animals, experimental drugs and experimental equipment with their creativity and innovation;

4) open network: Opening to network made the experimenter enter or exit the process freely.

5) Uses broad: It is easy for showing, preparing and conducting for teacher and students.

In addition, it also has chiropractics such as experimental low-cost, small difficulty, time less and small space restrictions, and user-friendly operation.

\section{Mentality Of Designing OF ViRTUAL}

\section{EXPERIMENTAL SYSTEM OF PHYSIOLOGICAL FUNCTION}

The virtual experiments should be designed with certain principles. Firstly, is it necessary? Secondly, what part should be designed and illustrated? It is necessary to consider that the human-computer interaction should have a simple operating interface, easy to facilitate and control. Moreover, the simulated experimental animals, instruments, equipment and experiments results should be vivid. It is better to use on-site environment and in-kind photo to display the data and the phenomenon. At last, it is necessary to consider whether the computer network teaching environment is suitable to carry out in specific school .The virtual experimental system can be designed in teaching application to reduce experimental costs and share teaching resource.

\section{STRUCTURE OF VIRTUAL EXPERIMENTAL SYSTEM OF PHYSIOLOGICAL FUNCTION}

\section{A. Software System}

To provide students with interactive learning function, the data with text, video and flash interactive carton can be get from the server based on the students' choice. Data management function and various virtual experiments of physiological function are supported by MySQL professional database. Client data access can be responded and transferred. The use of client / server architecture can be visited not only in the laboratory LAN, but also in campus network. It is easy for students to use, expand and update.

\section{B. System Architecture}

The system uses a server / client mode. The database were stored and managed on the server, The client reads material of the server, then displays to the user.

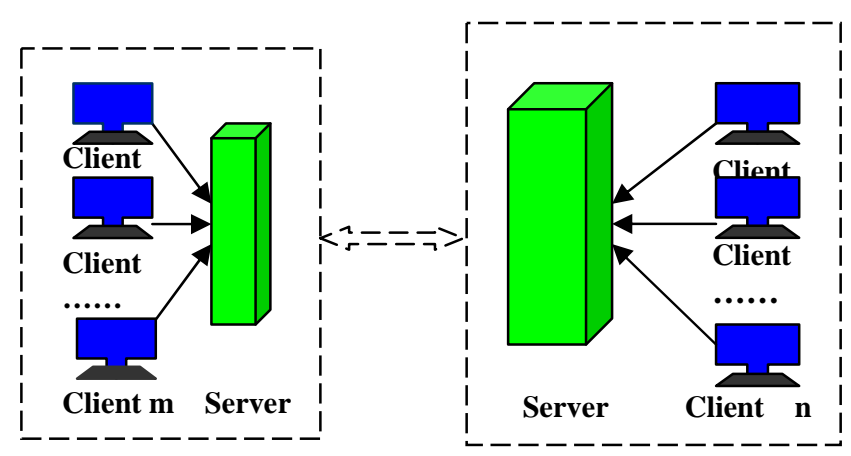

Figure 1. Overall structure diagram of virtual experimental system of physiological function

\section{The Structure of the Client}

The client is an interface between users and the system. The client is equivalent to a browser while requires and explains data from server.

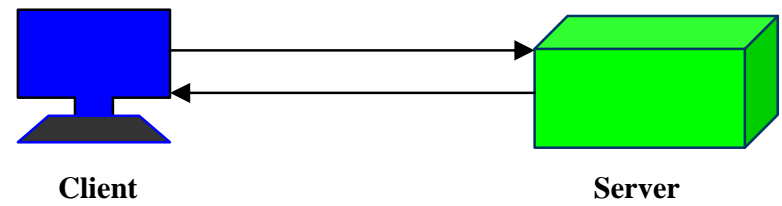

Figure2. Client structure diagram of virtual experimental system of physiological function

\section{Server Structure}

Server is the source of virtual experimental system data. It could offer and modify data. The server receives request from clients and selects data from database. Then the server returns data to corresponding clients. Updating data includes modifying, adding and testing data. The server offer interface for updating data. The data and access path can be updated. with this way, The testing data is based on information from database to test the availability of the resource

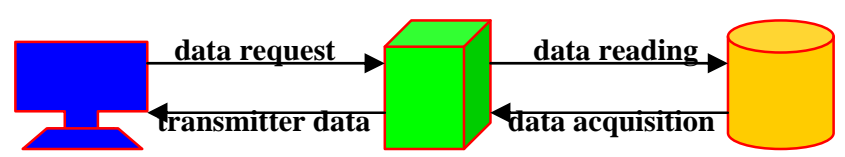

Client

Server

Database

Figure 3. Server structure diagram of virtual experimental system of physiological function 


\section{E. Functional Modules}

The functional modules of virtual system of physiological function experiments include the following components:

Experimental reference space which includes the physiological functional experiment. The common technique knowledge can be used to play video and explain experimental requirements. Laboratory animal space includes basic knowledge of a variety of experimental animals, laboratory animals, and the use of technology. Experimental preparation space, which includes items cabinet for storage of laboratory instruments, reagents and surgical instruments, can be used as experimental material, text, images and three-dimensional model similar with real lab laboratory. Simulation laboratory includes the corresponding experimental material to simulate the experimental procedure which has the test of drug and operations. Experiment results can be displayed after you finish corresponding operation. In addition, drug dose or the surgical procedure forgotten can be checked by watching the demo and video. Simulation examination space can be used to test students' ability to master preview and after-school knowledge with physiology preview questions and experimental examination. It can save a lot of manpower and time resources by paperless examination.

\section{RESEARCH AND DEVELOPMENT TRENDS OF VIRTUAL SYSTEM OF PHYSIOLOGICAL FUNCTION EXPERIMENTS}

Nowadays, modern information technology mainly based on computers and network technology makes teaching methods in higher education face unprecedented opportunities and challenges. Virtual experimental teaching system shows broad prospects for development as a new way of education. Virtual system of physiological function experiments is a innovation in the information age, a novel development of experimental lesson. It promote the teaching concept and ways. Virtual experimental of physiological function is playing unique advantages in physiological experiments lesson. It can prompt us to update experimental teaching concepts, build an open experimental teaching environment, increase physiology experiment lesson content, help students' self-learning, improve the ways and methods of experimental lesson, improve the quality of teaching and cultivate students' innovative spirit.

\section{REFERENCES}

[1] D.Z. Gong, Y.P. Sun, D.M. Wang. The development and application of computer network technology in functional laboratory teaching. Medical Education, 2003, (2) 40-41

[2] J.J.Zhang, H.X. Huang, C.C. Yan. Virtual platform design . Modern electronic technology 2004, (11) B40-44.

[3] W.T.Qiu. WEB-based virtual laboratory building. Journal of Distance Education 2002, (6) B39 - 42.
[4] Sh.F.Dai, B.B Zhang, L.H.Li. The medical function virtual laboratory in diversified experimental teaching. Medical information , 2010, ( 23 ) 4,849.

[5] Y.R.Zhang, K.Wu, X.M. Wu. Medical virtual instrument Network Laboratory based on the campus network. Medical equipment information, 2006 , ( 21) 5,25-27.

[6] Y. Han, X.Y. Gao, X.H. Qi, G.Q. Zhu. The establishment and application of virtual physiology lab. Medical education, 2005 (4) , $63-65$.

[7] J. Xu, Y.P. Sun, D.Mi.Wang . Comparison between Virtual experiment and real experiment in functional experiment Medical Education 2009, (30) 6,75-76.

[8] G.P. Lin, Q.L. Xiang, Sh.Zh.Wang, T.H.Wang . Virtual experiment and its application in teaching physiology. Modern Educational Technology , 2007 , ( 6 ) 10,948-955 . 